\title{
The role of glutamate excitotoxicity and neuroinflammation in depression and suicidal behavior: focus on microglia cells
}

\author{
Gianluca Serafini ${ }^{1}$, Zoltan Rihmer², Mario Amore ${ }^{1}$ \\ ${ }^{1}$ Department of Neuroscience, Rehabilitation, Ophthalmology, Genetics, Maternal and Child Health, Section of Psychiatry, \\ University of Genoa, 16126 Genova, Italy. \\ ${ }^{2}$ Department of Clinical and Theoretical Mental Health, Semmelweis University, H-1085 Budapest, Hungary.
}

\begin{abstract}
Major depressive disorder (MDD) is associated with a significant disability worldwide, relevant psychosocial impairment, and increased risk of suicidal behavior. ${ }^{[1]}$ Although multiple psychoactive compounds are now available, ${ }^{[2]}$ more than $20 \%$ of MDD patients treated with traditional antidepressant drugs do not benefit from complete recovery and are affected by treatment-resistance. ${ }^{[3]}$ Traditional antidepressant medications may be ineffective and sometimes worsen depressive symptoms in a vulnerable subpopulation of patients with subthreshold hypomanic symptoms that may be better included in the bipolar spectrum rather than MDD. ${ }^{[4]}$
\end{abstract}

The most recent years have been characterized by the paradigm shift from the monoamine conceptualization of depression to neuroplasticity hypothesis mainly focused on glutamatergic dysfunctions. ${ }^{[5]}$ There are consistent evidence reporting that abnormalities of glutamatergic neurotransmission are common in depressed individuals. ${ }^{[6]}$ Specifically, N-methyl-D-aspartate receptors (NMDAR) overactivation seems to play a critical role in the pathogenesis of MDD as reducing their functioning may be associated with mood recovery. For instance, ketamine has been recently investigated for its potential antidepressant effects ${ }^{[7]}$ and improvement of suicidal ideation ${ }^{[8]}$ beyond the monoamine hypothesis.

In addition, the existence of abnormalities in inflammatory processes in depressed patients suggests

\begin{tabular}{|l|l|}
\hline \multicolumn{3}{|c|}{ Access this article online } \\
\hline Quick Response Code: & \\
\hline & Website: \\
\hline & Www.nnjournal.net \\
& \\
\hline
\end{tabular}

the immunological origin of major depression. ${ }^{[9]}$ Inflammatory mediators and oxidative stress may lead to glutamate excitotoxicity playing a significant role in the pathogenesis of MDD. ${ }^{[10]}$ Notably, immunological differences have been frequently observed in patients with MDD and suicidal behavior.

Glial cells have been proposed as potential candidate targets for both glutamatergic-and inflammatory-mediated alterations underlying MDD and suicidal behavior. ${ }^{[11,12]}$ Historically, glial cells may be grouped in astrocytes, oligodendrocytes, and microglia.

Microglial cells derive from the immune system and may be considered the immunologic sentinel cells of the brain. As the activation of microglial cells was associated with the abnormal production of inflammatory mediators, ${ }^{[13,14]}$ these cells have been proposed as possible effectors of the abnormal immune response in MDD. They provide immunomodulatory functions, ${ }^{[15]}$ and functionally support neural plasticity-processes. ${ }^{[9]}$

Importantly, the abnormal activation of microglial cells reflected long-lasting depression- and anxiety-like behavioral effects. ${ }^{[11]}$ This has been also confirmed by the fact that minocycline with anti-inflammatory properties and microglia inactivation is not only able to reverse microglial alterations, ${ }^{[16]}$ but is also associated with antidepressant-like activity in rats exposed to learned helplessness. ${ }^{[17]}$

Unfortunately, some of the existing studies supporting the link between abnormal glial activation and MDD/ suicidal behavior are limited by small sample size. As an example, Bayer et al. ${ }^{[18]}$ in a postmortem study found elevated microglial cells in both frontal cortex and hippocampus of 6 depressed and 14 psychotic

Corresponding Author: Dr. Gianluca Serafini, Department of Neuroscience, Rehabilitation, Ophthalmology, Genetics, Maternal and Child Health, Section of Psychiatry, University of Genoa, IRCCS San Martino, Largo Rosanna Benzi 10, 16126 Genova, Italy. E-mail: gianluca.serafini@unige.it 
individuals compared to 13 healthy controls. Steiner et al. ${ }^{[19]}$ observed increased microglial cell numbers in the anterior cingulate cortex and mediodorsal thalamus of two individuals who committed suicide during acute psychosis. However, no effect of diagnosis on the microglial density but only significant microgliosis was reported in dorsolateral prefrontal cortex, anterior cingulate cortex, mediodorsal thalamus, and hippocampus of 16 schizophrenic and 14 depressed subjects died by suicide. ${ }^{[20]}$ The authors hypothesized that the link between microglial activation and suicidal behavior may be mediated by neuroendocrine factors such as inflammatory cytokines and oxidative stress. Finally, Dean et al. ${ }^{[21]}$ showed that CD11b (a potential microglia/macrophages marker) was not increased in the cortex of ten subjects with MDD, and ten with bipolar disorder.

To the best of our knowledge, there are no reports in the current literature concerning the association between microglial glutamatergic abnormalities and MDD/suicidal behavior with the exception of the review of Niciu et al. ${ }^{[22]}$ suggesting that glial-mediated glutamatergic dysfunction is a common neuropathological pathway in patients with substance use disorders and MDD.

It is currently unclear whether microglial abnormal activation may directly induce psychopathological conditions, should be considered an epiphenomenon of other related processes associated, in turn, with psychopathological conditions, or alternatively a nonspecific tissue reaction independent of psychopathology. Another controversial issue concerns the exact relationship between inflammatory stressful stimuli, autoimmunity, and abnormalities in glutamatergic activity.

The sequence of molecular events underlying MDD and suicidal behavior is still poorly understood. Microglia and monocytes are usually involved in the integration of sensory information within the peripheral sensory nerves and endocrine system. ${ }^{[23]}$ Stress and other signaling molecules (e.g. cytokines, oxidative free radicals) may activate the oxidation sensitive

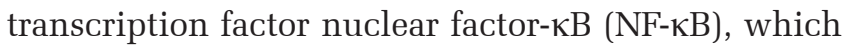
is highly expressed in microglia with the final result of increased NF- $\mathrm{KB}$-DNA binding and transcription of genes encoding for chemokines, cytokines, and oxidases/proteases. As suggested by the same authors, ${ }^{[24]}$ microglia reported morphological changes in response to exposure to both environmental and internal stimuli.

Furthermore, antibodies against serotonin have been commonly found in more than $50 \%$ of depressed patients and importantly, in all those conditions in which increased inflammatory cytokines were observed. ${ }^{[24]}$ They cannot affect brain functions until inflammatory mediators do alter the integrity of blood-brain barrier. However, when a blood-brain barrier alteration occurs, antibodies may presumably cross-react with the subunits of NMDAR on glial cells inducing the abnormal release of glutamate. This enhanced activation of glial cells is associated with glutamatergic excitotoxicity, apoptosis, and clinically significant behavioral changes. ${ }^{[25,26]}$ Also, as suggested by Santello et al., ${ }^{[27]}$ tumor necrosis factor alpha (TNF- $\alpha$ ) controls the neuromodulatory action of dentate granule cell synapses in astrocytes, through $\mathrm{Ca}^{2+}$-dependent glutamate release and pre-NMDAR activation. Therefore, gliotransmission together with its synaptic effects seem to be controlled not only by astrocyte $\mathrm{Ca}^{2+}$ elevations but also by homeostatic factors such as TNF- $\alpha$. Previous studies have shown that both the excitatory neurotransmitter glutamate and the proinflammatory cytokine TNF- $\alpha$ may be considered as effectors of microglial-stimulated death. ${ }^{[28]}$

Recently, Schnieder et al. ${ }^{[29]}$ also found a $18 \%$ greater density of perivascular cells in dorsal white matter prefrontal cortex of 11 subjects died by suicide suggesting the induction of important alterations in the characteristics of blood-brain barrier in microglia cells of these individuals. Autoimmune activity directed against serotonin may directly compromise serotonergic axons and their functioning with the final result of relevant deficits in serotonergic neurotrasmission. Serotonin may be disrupted by abnormal (e.g. hyperactivated) pathways such as that of kynurenine inside microglial cells due to the enhanced metabolism of tryptophan to quinolinic acid. ${ }^{[30,31]}$

Elevated levels of kynurenic acid, an astrocyte-derived metabolite of the kynurenine pathway has been reported to significantly reduce glutamate release in some brain regions such as the hippocampus. ${ }^{[32,33]}$ Also, increased micromolar levels of kynurenic acid have been suggested to inhibit a-amino-3-hydroxy-5-methyl-4-isoxazole proprionic acid and kainate receptors. ${ }^{[34,35]}$

Inflammatory cytokines may further induce the abnormal release of quinolinic acid in microglia ${ }^{[36]}$ related to aberrant stimulation of neurons in the ventral prefrontal cortex and altered connectivity between cortical structures and limbic system.

The critical role of quinolinic acid in microglia-abnormal activation has been also demonstrated by the elevated levels of indoleamine 2,3-dioxygenase and kynurenine monooxygenase in the quinolinic acid biosynthesis pathway in CX3CR1 knockout mice. ${ }^{[37]}$ Notably, recent compounds with antidepressant properties 
such as ketamine ${ }^{[38]}$ as well as selective serotonin reuptake inhibitors ${ }^{[39]}$ and tricyclic ${ }^{[40,41]}$ may reverse the neurodegenerative activation of microglia induced by pathologically increased inflammatory cytokines.

Furthermore, increased glucocorticoids levels may affect the integrity of microglial cells in the early phases of depression. ${ }^{[42]}$ Abnormal extracellular glutamate concentrations may spill into microglial cells exerting neurotoxic effects on $\gamma$-aminobutyric acid neurons. ${ }^{[43]}$ The uptake of glutamate is progressively decreased, and the density of glutamatergic pyramidal neurons is reduced in depressed individuals ${ }^{[44]}$ with the final result of reduced cortical levels of glutamate in the later phases of depression.

Overall, microglial cells are able to exert significant immunomodulatory functions in the central nervous system. Structural changes induced by chronic stress and MDD on glial cells may contribute to the pathophysiology of these conditions, but they may be significantly reversed using modern antidepressant medications. Aberrant levels of quinolinic acid produced by abnormal pathways inside microglia cells represent a valid intracellular mediator of pathologically inflammatory- and glutamatergic-related changes. A complex interaction between dysfunctional inflammatory pathways, increased oxidative stress, altered neuroplasticity in glial cells and neuronal abnormalities are involved in both MDD and suicidal behavior.

\section{REFERENCES}

1. Greer TL, Kurian BT, Trivedi MH. Defining and measuring functional recovery from depression. CNS Drugs 2010;24:267-84.

2. Serafini G, Pompili M, Innamorati M, Dwivedi Y, Brahmachari G, Girardi P. Pharmacological properties of glutamatergic drugs targeting NMDA receptors and their application in major depression. Curr Pharm Des 2013;19:1898-922.

3. Trivedi MH, Rush AJ, Wisniewski SR, Nierenberg AA, Warden D, Ritz L, Norquist G, Howland RH, Lebowitz B, McGrath PJ, Shores-Wilson K, Biggs MM, Balasubramani GK, Fava M. Evaluation of outcomes with citalopram for depression using measurement-based care in STAR*D: implications for clinical practice. Am J Psychiatry 2006;163:28-40.

4. Rihmer Z, Gonda X. Antidepressant-resistant depression and antidepressant-associated suicidal behaviour: the role of underlying bipolarity. Depress Res Treat 2011;2011:906462.

5. Sanacora G, Treccani G, Popoli M. Towards a glutamate hypothesis of depression: an emerging frontier of neuropsychopharmacology for mood disorders. Neuropharmacology 2012;62:63-77.

6. Yüksel C, Öngür D. Magnetic resonance spectroscopy studies of glutamate-related abnormalities in mood disorders. Biol Psychiatry 2010;68:785-94.

7. Niciu MJ, Luckenbaugh DA, Ionescu DF, Guevara S, Machado-Vieira R, Richards EM, Brutsche NE, Nolan NM, Zarate CA Jr. Clinical predictors of ketamine response in treatment-resistant major depression. J Clin Psychiatry 2014;75:e417-23.

8. Ballard ED, Ionescu DF, Vande Voort JL, Niciu MJ, Richards EM, Luckenbaugh DA, Brutsche NE, Ameli R, Furey ML, Zarate CA
Jr. Improvement in suicidal ideation after ketamine infusion: relationship to reductions in depression and anxiety. $J$ Psychiatr Res 2014;58:161-6.

9. Schafer DP, Lehrman EK, Kautzman AG, Koyama R, Mardinly AR, Yamasaki R, Ransohoff RM, Greenberg ME, Barres BA, Stevens B. Microglia sculpt postnatal neural circuits in an activity and complement-dependent manner. Neuron 2012;74:691-705.

10. Serafini G, Pompili M, Elena Seretti M, Stefani H, Palermo M, Coryell W, Girardi P. The role of inflammatory cytokines in suicidal behavior: a systematic review. Eur Neuropsychopharmacol 2013;23:1672-86.

11. Frick LR, Williams K, Pittenger C. Microglial dysregulation in psychiatric disease. Clin Dev Immunol 2013;2013:608654.

12. Blank T, Prinz M. Microglia as modulators of cognition and neuropsychiatric disorders. Glia 2013;61:62-70.

13. Frank MG, Baratta MV, Sprunger DB, Watkins LR, Maier SF. Microglia serve as a neuroimmune substrate for stress-induced potentiation of CNS pro-inflammatory cytokine responses. Brain Behav Immun 2007;21:47-59.

14. Hanisch UK. Microglia as a source and target of cytokines. Glia 2002;40:140-55.

15. David S, Kroner A. Repertoire of microglial and macrophage responses after spinal cord injury. Nat Rev Neurosci 2011;12:388-99.

16. Hinwood M, Morandini J, Day TA, Walker FR. Evidence that microglia mediate the neurobiological effects of chronic psychological stress on the medial prefrontal cortex. Cereb Cortex 2012;22:1442-54.

17. Arakawa S, Shirayama Y, Fujita Y, Ishima T, Horio M, Muneoka K, Iyo M, Hashimoto K. Minocycline produced antidepressant-like effects on the learned helplessness rats with alterations in levels of monoamine in the amygdala and no changes in BDNF levels in the hippocampus at baseline. Pharmacol Biochem Behav 2012;100:601-6.

18. Bayer TA, Buslei R, Havas L, Falkai P. Evidence for activation of microglia in patients with psychiatric illnesses. Neurosci Lett 1999;271:126-8.

19. Steiner J, Mawrin C, Ziegeler A, Bielau H, Ullrich O, Bernstein HG, Bogerts B. Distribution of HLA-DR-positive microglia in schizophrenia reflects impaired cerebral lateralization. Acta Neuropathol 2006;112:305-16.

20. Steiner J, Bielau H, Brisch R, Danos P, Ullrich O, Mawrin C, Bernstein HG, Bogerts B. Immunological aspects in the neurobiology of suicide: elevated microglial density in schizophrenia and depression is associated with suicide. J Psychiatr Res 2008;42:151-7.

21. Dean B, Gibbons AS, Tawadros N, Brooks L, Everall IP, Scarr E. Different changes in cortical tumor necrosis factor- $\alpha$-related pathways in schizophrenia and mood disorders. Mol Psychiatry 2013;18:767-73.

22. Niciu MJ, Henter ID, Sanacora G, Zarate CAJr. Glial abnormalities in substance use disorders and depression: does shared glutamatergic dysfunction contribute to comorbidity? World J Biol Psychiatry 2014;15:2-16.

23. Crews FT, Vetreno RP. Addiction, adolescence, and innate immune gene induction. Front Psychiatry 2011;2:19.

24. Maes M, Fišar Z, Medina M, Scapagnini G, Nowak G, Berk M. New drug targets in depression: inflammatory, cell-mediated immune, oxidative and nitrosative stress, mitochondrial, antioxidant, and neuroprogressive pathways. And new drug candidates - Nrf2 activators and GSK-3 inhibitors. Inflammopharmacology 2012;20:127-50.

25. Diamond B, Volpe BT. A model for lupus brain disease. Immunol Rev 2012;248:56-67.

26. Faust TW, Chang EH, Kowal C, Berlin R, Gazaryan IG, Bertini E, Zhang J, Sanchez-Guerrero J, Fragoso-Loyo HE, Volpe BT, Diamond B, Huerta PT. Neurotoxic lupus autoantibodies alter brain function through two distinct mechanisms. Proc Natl Acad Sci U S A 2010;107:18569-74.

27. Santello M, Bezzi P, Volterra A. TNF $\alpha$ controls glutamatergic gliotransmission in the hippocampal dentate gyrus. Neuron 2011;69:988-1001. 
28. Floden AM, Li S, Combs CK. Beta-amyloid-stimulated microglia induce neuron death via synergistic stimulation of tumor necrosis factor alpha and NMDA receptors. $J$ Neurosci 2005;25:2566-75.

29. Schnieder TP, Trencevska I, Rosoklija G, Stankov A, Mann JJ, Smiley J, Dwork AJ. Microglia of prefrontal white matter in suicide. J Neuropathol Exp Neurol 2014;73:880-90.

30. Myint AM, Kim YK. Cytokine-serotonin interaction through IDO: a neurodegeneration hypothesis of depression. Med Hypotheses 2003;61:519-25.

31. Hu B, Hissong BD, Carlin JM. Interleukin-1 enhances indoleamine 2, 3-dioxygenase activity by increasing specific mRNA expression in human mononuclear phagocytes. J Interferon Cytokine Res 1995; 15:617-24.

32. Carpenedo R, Pittaluga A, Cozzi A, Attucci S, Galli A, Raiteri M, Moroni F. Presynaptic kynurenate-sensitive receptors inhibit glutamate release. Eur J Neurosci 2001;13:2141-7.

33. Pocivavsek A, Wu HQ, Potter MC, Elmer GI, Pellicciari R, Schwarcz R. Fluctuations in endogenous kynurenic acid control hippocampal glutamate and memory. Neuropsychopharmacology 2011;36:2357-67.

34. Stone TW. Neuropharmacology of quinolinic and kynurenic acids. Pharmacol Rev 1993;45:309-79.

35. Moroni F. Tryptophan metabolism and brain function: focus on kynurenine and other indole metabolites. Eur J Pharmacol 1999;375:87-100.

36. Guillemin GJ. Quinolinic acid, the inescapable neurotoxin. FEBS J 2012;279:1356-65.

37. Corona AW, Huang Y, O'Connor JC, Dantzer R, Kelley KW, Popovich PG, Godbout JP. Fractalkine receptor (CX3CR1) deficiency sensitizes mice to the behavioral changes induced by lipopolysaccharide. J Neuroinflammation 2010;7:93.
38. Chang Y, Lee JJ, Hsieh CY, Hsiao G, Chou DS, Sheu JR. Inhibitory effects of ketamine on lipopolysaccharide-induced microglial activation. Mediators Inflamm 2009;2009:705379.

39. Liu D, Wang Z, Liu S, Wang F, Zhao S, Hao A. Anti-inflammatory effects of fluoxetine in lipopolysaccharide (LPS)-stimulated microglial cells. Neuropharmacology 2011;61:592-9.

40. Obuchowicz E, Kowalski J, Labuzek K, Krysiak R, Pendzich J, Herman ZS. Amitriptyline and nortriptyline inhibit interleukin-1 release by rat mixed glial and microglial cell cultures. Int $J$ Neuropsychopharmacol 2006;9:27-35.

41. Liu Q, Li B, Zhu HY, Wang YQ, Yu J, Wu GC. Clomipramine treatment reversed the glial pathology in a chronic unpredictable stress-induced rat model of depression. Eur Neuropsychopharmacol 2009;19:796-805.

42. Rajkowska G, Miguel-Hidalgo JJ. Gliogenesis and glial pathology in depression. CNS Neurol Disord Drug Targets 2007;6:219-33.

43. Rajkowska G, O'Dwyer G, Teleki Z, Stockmeier CA Miguel-Hidalgo JJ. GABAergic neurons immunoreactive for calcium binding proteins are reduced in the prefrontal cortex in major depression. Neuropsychopharmacology 2007;32:471-82.

44. Rajkowska G, Miguel-Hidalgo JJ, Dubey P, Stockmeier CA, Krishnan KR. Prominent reduction in pyramidal neurons density in the orbitofrontal cortex of elderly depressed patients. Biol Psychiatry 2005;58:297-306.

Cite this article as: Serafini G, Rihmer Z, Amore M. The role of glutamate excitotoxicity and neuroinflammation in depression and suicidal behavior: focus on microglia cells. Neuroimmunol Neuroinflammation 2015;2(3):127-30. Source of Support: Nil. Conflict of Interest: No.

Received: 25-09-2014; Accepted: 18-01-2015 